\title{
Classification of Sonar Targets in Air-A Neural Network Approach ${ }^{+}$
}

\author{
Patrick K. Kroh ${ }^{1, *}$, Ralph Simon ${ }^{2}$ and Stefan J. Rupitsch ${ }^{1}$ \\ 1 Lehrstuhl für Sensorik, Friedrich-Alexander-Universität Erlangen, 91052 Erlangen, Germany; \\ stefan.rupitsch@fau.de \\ 2 Department of Ecological Science, Vrije Universiteit Amsterdam, 1081 HV Amsterdam, The Netherlands; \\ ralph.simon@vu.nl \\ * Correspondence: patrick.k.kroh@fau.de; Tel.: +49-9131-85-23149 \\ † Presented at the Eurosensors 2018 Conference, Graz, Austria, 9-12 September 2018.
}

Published: 29 November 2018

\begin{abstract}
Ultrasonic sonar sensors are commonly used for contactless distance measurements in application areas such as automotive and mobile robotics. They can also be used to identify and classify sound reflecting objects. In the presented work, we classify simple sonar targets of different geometric shape and size. For this purpose, we built a test stand for echo measurements that facilitates defined arbitrary translation and rotation of the targets. Artificial neural networks (ANNs) with multiple hidden layers were used as classifiers and different features were evaluated. The focus was on two features that were derived from the echoes' cross-correlation functions with their excitation chirp signals. We could distinguish different target geometries with our features and also evaluated the ANNs' capabilities for size discrimination of targets with the same geometric shape.
\end{abstract}

Keywords: sonar measurements; sonar detection; neural networks; feature extraction

\section{Introduction}

Air-based ultrasonic sonar sensors are often used for obstacle avoidance and navigation purposes in application areas such as automotive, factory automation as well as mobile ground and airborne robotics [1]. In addition to identifying the distance to the closest obstacle, it is also favorable to be able to classify targets, which may then be used as landmarks for navigation and positioning. We used broadband chirp signals-contrary to most other sonar applications-as their crosscorrelation functions are narrow and they are better suited to deduce object features. Besides, echolocating bats are known to use broadband signals as soon as they have to resolve objects in front of vegetation [2] and it was also shown that they are able to classify different geometrical objects independent of their size [3]. As classifiers, ANNs are an option, which were used for ultrasonic targets in air [4] and for classification of spherical targets consisting of different materials in water [5]. In our work, we focus on classification of geometrically different shaped targets and the extraction of suitable sonar features.

\section{Targets}

We obtained and characterized ultrasound echoes from six different targets. Those were: two flat discs (30 $\mathrm{mm}$ and $50 \mathrm{~mm}$ radii, $4 \mathrm{~mm}$ thickness), two cylinders ( $30 \mathrm{~mm}$ and $50 \mathrm{~mm}$ radii, $60 \mathrm{~mm}$ and $100 \mathrm{~mm}$ heights, respectively) and two concave hemispheres ( $30 \mathrm{~mm}$ and $50 \mathrm{~mm}$ radii, $2 \mathrm{~mm}$ wall thickness). The three different geometric shapes have got characteristic acoustic fingerprintsspectrum versus rotation angle plots (see Figure 1). Hence, they should be well-distinguishable and 
were thus chosen. Detailed explanations regarding the target geometries' acoustic properties can be found in [6].

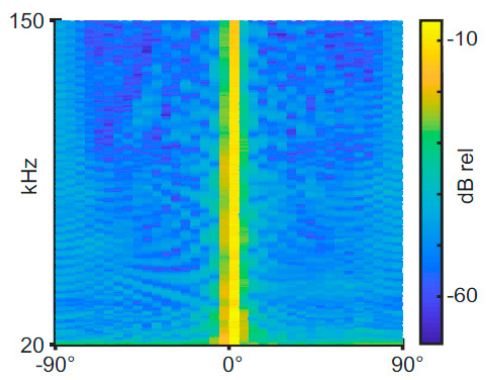

(a) Disc, $50 \mathrm{~mm}$ radius

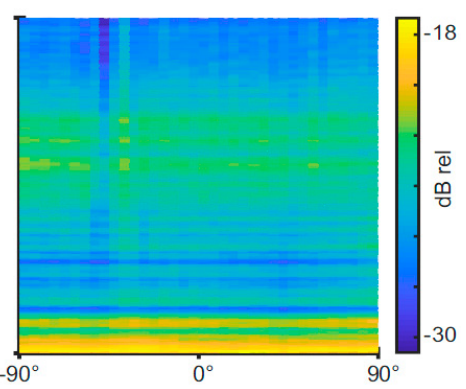

(b) Cylinder, $50 \mathrm{~mm}$ radius, $100 \mathrm{~mm}$ height

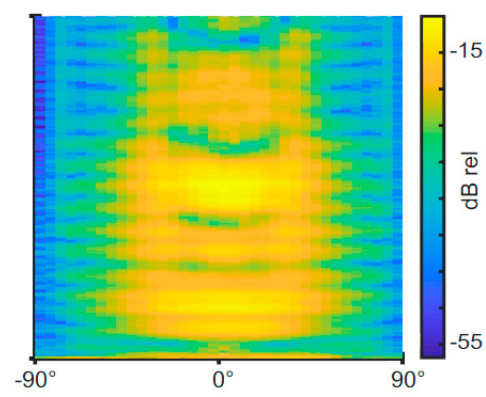

(c) Concave hemisphere, $50 \mathrm{~mm}$ radius

Figure 1. Echo fingerprint for different target geometric shapes at a distance of $1 \mathrm{~m}$, relative to speaker frequency characteristic at a distance of $1 \mathrm{~m}$, measured with single-frequency sine bursts.

\section{Measurement Setup}

All measurements were performed in an anechoic chamber. The measurement setup (as shown in Figure 2) consisted of a two axis translation stage, a rotation stage on top of the translation stage on which the targets were attached, a 1/4" Bruel\&Kjaer measurement microphone (Type 4939-A-011) with an amplifier (G.R.A.S. 12AK), a wideband electrostatic ultrasonic speaker (Senscomp 7000 series) with a custom-built high voltage amplifier ( $0 \mathrm{~V}$ to $400 \mathrm{~V}, 0 \mathrm{kHz}$ to $200 \mathrm{kHz}$ ) and a National Instruments data acquisition device for analog IO (NI-USB-6356, 1.25 MSa/s, 16 bit). The microphone and the speaker were mounted closely together (20 mm center distance) at the end of the $x$-axis translation stage. The targets, microphone and speaker were located $1 \mathrm{~m}$ above the floor. The setup was optimized so that there was no detectable direct echo from itself. Echoes that resulted from multiple reflections, appeared after the target echoes in the measured waveforms.

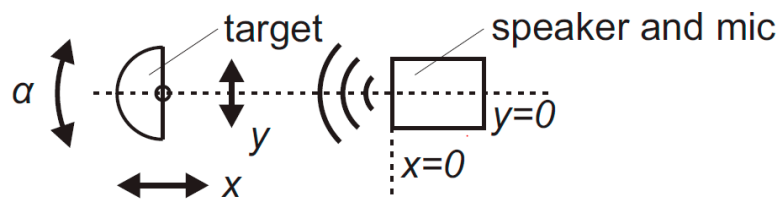

Figure 2. Measurement setup.

\section{Measurement Procedure}

For the ANN training and test data, the targets were automatically moved along a grid and also rotated-x-direction $(0.5 \mathrm{~m}$ to $1.8 \mathrm{~m}, 0.1 \mathrm{~m}$ steps); $\mathrm{y}$-direction $(-0.15 \mathrm{~m}$ to $0.15 \mathrm{~m}, 0.05 \mathrm{~m}$ steps); angles $\left(-60^{\circ}\right.$ to $60^{\circ}, 15^{\circ}$ steps). We used downward modulated wideband chirp signals ( $150 \mathrm{kHz}$ to $20 \mathrm{kHz}$, $1 \mathrm{~ms}$ duration) - referred to as $w b$. Additionally, we also used narrowband chirp signals ( $n b, 52 \mathrm{kHz}$ to $48 \mathrm{kHz}, 1 \mathrm{~ms}$ duration) as we were also interested in the performance that may be achieved if we used a common narrowband ultrasonic sensor, such as a piezoelectric-based transducer. Chirp signals were chosen as they make it possible to gain information regarding a large portion of the spectrum from a single echo. From the complete recorded echoes, we selected a region of interest (ROI) of $3 \mathrm{~ms}$ for our feature calculations. The ROI positions were determined by the sound propagation distance to the target, which is known in this case. We also obtained true negative samples-non-target echoes-for proper ANN training. For this purpose, we added additional random time to the targets' propagation delays for the non-target ROIs, in which echoes from multiple reflections off the measurement setup appear. 


\section{Neural Networks and Feature Engineering}

We designed our ANNs with four hidden layers in order to achieve good generalization and to avoid overfitting to the training data. The hidden layers comprised 10, 5, 5 and 3 neurons, respectively. A scaled conjugate gradient backpropagation algorithm with a cross entropy error function was used for training. For more information on ANNs, see [7]. We performed supervised learning as we knew the target positions as well as the target classes from the measurement procedure and could, thus, create labeled data sets.

Tests with various features were performed, of which two are of special interest. The first of these features is the spectrogram of an echo's cross-correlation function with its corresponding excitation chirp signal-referred to as FSP. For each target position, the according ROI was selected from the spectrogram. The resulting $2 \mathrm{D}$ array that contained the values, was flattened into a $1 \mathrm{D}$ array which was included into the final feature vector. The second feature is the logarithmic maximum value of the previously mentioned cross-correlation function, with an additional term to compensate for spherical spreading loss, which is based on the sonar equation-referred to as FTS. This feature is proportional to a rough estimate of a reflector's target strength. In addition, we have also combined FSP and FTS-referred to as FC.

A feature vector was chosen to consist of one of the features for five neighboring measurement grid positions. This is equivalent to a sonar sensor traveling by a target and taking five measurements. We have evaluated our ANNs by analyzing the confusion matrices and have deduced precision as well as recall values from those matrices. Precision and recall values are to be maximized towards $100 \%$ and are calculated for each target class as given:

$$
\begin{aligned}
& \text { Precision }=\frac{\text { no(true positives })}{\text { no(false positives })+ \text { no(true positives })} \\
& \text { Recall }=\frac{\text { no(true positives })}{\text { no(false negatives })+ \text { no(true positives })}
\end{aligned}
$$

\section{Classification Results}

Results for precision and recall are summarized in Table 1. It can clearly be seen that our ANNs are able to classify the general geometric target shapes in most cases. Apart from that, good classification among different reflector sizes seems to be possible as well. Size discrimination of the hemispheres works best. Most ambiguous classifications can be observed for disc shaped targets. We assume that this happens if a disc is rotated in a way that only a weak echo is reflected back-see Figure 1 for comparison. In order to determine whether this is actually the case, further investigation is planned and may lead to optimization opportunities for good performance with all target shapes and sizes. The FTS can be used alone as well, but yields a lower performance in contrast to the spectrogram features, which show the best results at the cost of larger computational effort. Use of FC, the combination of FSP and FTS, does not seem to lead to further significantly improved performance. Also, narrowband signals were tested successfully, hence the use of piezoelectric transducers may be possible in the future.

Classification was performed with a well-defined set of targets and true negatives that can be separated from the targets well. It thus remains to be investigated how robustly the system will perform if echoes from cluttered spaces are included, which may often be the case for applications outside the lab space. In this context, also the separation of echoes from multiple targets in close distance to each other is of interest and may require the inclusion of sophisticated sound source localization techniques. Here, an additional opportunity for improvement is to engineer and test more features in combination with feature evaluation tools such as the principal component analysis, which will presumably lead to a reduction of the necessary computational effort for ANN training and employment in comparison to the use of large spectrogram feature vectors. 
Table 1. Comparison of precision and recall for different signals and features; format: (precision in \%, recall in \%), wb: wideband, nb: narrowband.

\begin{tabular}{|c|c|c|c|c|c|c|c|}
\hline Shape & No Target & \multicolumn{2}{|c|}{ Disc } & \multicolumn{2}{|c|}{ Cylinder } & \multicolumn{2}{|c|}{ Hemisphere } \\
\hline wb, FSP & $98.8,100$ & \multicolumn{2}{|c|}{$94.5,74.1$} & \multicolumn{2}{|c|}{$84.6,92.5$} & \multicolumn{2}{|c|}{$91.5,100$} \\
\hline nb, FSP & $97.6,98.8$ & \multicolumn{2}{|c|}{$96.1,90.0$} & \multicolumn{2}{|c|}{$99.2,100$} & \multicolumn{2}{|c|}{$98.3,100$} \\
\hline wb, FTS & $96.2,98.6$ & \multicolumn{2}{|c|}{$95.3,87.0$} & \multicolumn{2}{|c|}{$88.8,97.9$} & \multicolumn{2}{|c|}{$97.0,98.1$} \\
\hline $\mathrm{nb}, \mathrm{FSP}$ & $94.1,96.7$ & \multicolumn{2}{|c|}{$81.9,70.8$} & \multicolumn{2}{|c|}{$67.1,97.0$} & \multicolumn{2}{|c|}{$96.3,63.6$} \\
\hline wb, FTS & $99.7,99.7$ & \multicolumn{2}{|c|}{$97.1,98.0$} & \multicolumn{2}{|c|}{$98.3,97.4$} & \multicolumn{2}{|c|}{$99.1,99.1$} \\
\hline nb, FSP & $99.7,98.6$ & \multicolumn{2}{|c|}{$95.8,97.5$} & \multicolumn{2}{|c|}{$98.2,95.0$} & \multicolumn{2}{|c|}{$94.0,100$} \\
\hline dim. & - & $\mathrm{r}=30 \mathrm{~mm}$ & $\mathrm{r}=50 \mathrm{~mm}$ & $\mathrm{r}=30 \mathrm{~mm}$ & $\mathrm{r}=50 \mathrm{~mm}$ & $\mathrm{r}=30 \mathrm{~mm}$ & $\mathrm{r}=50 \mathrm{~mm}$ \\
\hline wb, FSP & $98.8,100$ & $83.3,70.2$ & $95.3,69.5$ & $73.1,92.5$ & $100,92.6$ & $98.2,100$ & $86.3,100$ \\
\hline $\mathrm{nb}, \mathrm{FSP}$ & $97.6,98.8$ & $91.3,76.4$ & $80.7,83.6$ & $98.3,93.4$ & $93.5,100$ & $96.3,100$ & 100,100 \\
\hline wb, FTS & $96.2,98.6$ & $69.6,27.1$ & $54.2,78.9$ & $93.5,95.6$ & $85.2,100$ & $93.8,96.8$ & $100,77.1$ \\
\hline nb, FSP & $94.1,96.7$ & $43.3,25.5$ & $49.1,57.8$ & $61.2,98.1$ & $75.9,95.7$ & $85.7,29.0$ & $72.9,72.9$ \\
\hline wb, FTS & $99.7,99.7$ & $84.4,77.6$ & $82.8,90.6$ & $98.2,90.3$ & $91.4,98.1$ & $98.2,98.2$ & 100,100 \\
\hline $\mathrm{nb}, \mathrm{FSP}$ & $99.7,98.6$ & $82.8,84.2$ & $79.0,80.3$ & $100,89.5$ & $96.9,100$ & 100,100 & $90.3,100$ \\
\hline
\end{tabular}

Author Contributions: Conceptualization, P.K.K.; Methodology, P.K.K.; Software, P.K.K.; Validation, P.K.K., R.S. and S.J.R.; Formal Analysis, P.K.K.; Investigation, P.K.K.; Resources, P.K.K. and R.S.; Data Curation, P.K.K.; Writing-Original Draft Preparation, P.K.K.; Writing-Review \& Editing, P.K.K., R.S. and S.J.R.; Visualization, P.K.K.; Supervision, S.J.R.; Project Administration, S.J.R.; Funding Acquisition, S.J.R.

Funding: This research received no external funding.

Acknowledgments: We especially thank Hans-Michael Günther from the "Lehrstuhl für Sensorik" in Erlangen. He provided practical electronics support as well as designed and built the high-voltage amplifier.

Conflicts of Interest: The authors declare no conflict of interest.

\section{References}

1. Kleeman, L.; Kuc, R. Sonar Sensing. In Springer Handbook of Robotics; Siciliano, B., Khatib, O., Eds.; Springer: Berlin, Germany, 2008; pp. 491-519, ISBN 978-3-540-30301-5.

2. Siemers, B.M.; Schnitzler, H.U. Echolocation signals reflect niche differentiation in five sympatric congeneric bat species. Nature 2004, 657-661, doi:10.1038/nature02547.

3. Von Helversen, D. Object classification by echolocation in nectar feeding bats: Size-independent generalization of shape. J. Comp. Physiol. A 2004, 515-521, doi:10.1007/s00359-004-0492-9.

4. Barshan, B.; Ayrulu, B.; Utete, S.W. Neural network-based target differentiation using sonar for robotics applications. IEEE Trans. Robot. Autom. 2000, 435-442, doi:10.1109/70.864239.

5. Dmitrieva, M.; Valdenegro-Toro, M.; Brown, K.; Heald, G.; Lane, D. Object classification with convolution neural network based on the time-frequency representation of their echo. In Proceedings of the IEEE 27th International Workshop on Machine Learning for Signal Processing (MLSP), Tokyo, Japan, 25-28 September 2017, doi:10.1109/MLSP.2017.8168134

6. Simon, R.; Holderied, M.W.; Von Helversen, O. Size discrimination of hollow hemispheres by echolocation in a nectar feeding bat. J. Exp. Biol. 2006, 3599-3609, doi:10.1242/jeb.02398.

7. Bishop, C.M. Pattern Recognition and Machine Learning; Jordan, M., Kleinberg, J., Scholkopf, B., Eds.; Springer: New York, NY, USA, 2006; ISBN 978-0387-31073-2.

(C) 2018 by the authors. Licensee MDPI, Basel, Switzerland. This article is an open access article distributed under the terms and conditions of the Creative Commons Attribution (CC BY) license (http://creativecommons.org/licenses/by/4.0/). 